\title{
IMPROVING SYNTHETIC APERTURE IMAGE BY IMAGE COMPOUNDING IN BEAMFORMING PROCESS
}

\author{
Oscar Martínez-Graullera ${ }^{1}$, Ricardo T. Higuti ${ }^{2}$, Carlos J. Martín ${ }^{1}$, Luis. G. Ullate ${ }^{1}$, \\ David Romero $^{1}$, and Montserrat Parrilla ${ }^{1}$ \\ ${ }^{1}$ Centro de Acústica Aplicada y Evaluación No Destructiva - UPM-CSIC. Ctra. de \\ Campo Real, Km 0,200. Arganda del Rey. Madrid. E-28500, Spain \\ ${ }^{2}$ Universidade Estadual Paulista, Departament of Electrical Engineering, Av Brasil, \\ 56, Ilha Solteira, SP, 15385-000, Brazil
}

\begin{abstract}
In this work, signal processing techniques are used to improve the quality of image based on multi-element synthetic aperture techniques. Using several apodization functions to obtain different side lobes distribution, a polarity function and a threshold criterium are used to develop an image compounding technique. The spatial diversity is increased using an additional array, which generates complementary information about the defects, improving the results of the proposed algorithm and producing high resolution and contrast images. The inspection of isotropic plate-like structures using linear arrays and Lamb waves is presented. Experimental results are shown for a 1-mm-thick isotropic aluminum plate with artificial defects using linear arrays formed by 30 piezoelectric elements, with the low dispersion symmetric mode S0 at the frequency of $330 \mathrm{kHz}$.
\end{abstract}

KEYWORDS: Ultrasonic Imaging, Lamb Waves, Synthetic Aperture Imaging, Image Compounding

PACS: $43.35 . \mathrm{ZC}$, 43.60.FG

\section{INTRODUCTION}

Structural health monitoring of plate-like structures is an important issue in many areas such as in the aerospace and energy industries. The structure is permanently inspected to detect impact damages, hidden corrosion and delaminations along the material lifecycle, and that must be detected in early damage stages due to safety reasons. Among the available techniques, ultrasonic guided waves are very effective because of its rapid and long-range inspection, and the possibility to embed sensors in the structure [1].

The increasing use of arrays in non-destructive evaluation (NDE) is also present in ultrasonic guided wave inspection, and various array geometries and signal processing techniques have been employed and evaluated to obtain good defect detection and sizing [2, 3, 4]. As the changes in the inspected structure are relatively slow, high imaging rates are not necessary, and synthetic aperture techniques (SA) can be employed. These techniques are mainly based on a simple data acquisition system and a powerful post-processing system where different beamforming strategies are applied to increase signal-to-noise ratio and resolution in

Review of Progress in Quantitative Nondestructive Evaluation, Volume 30

AIP Conf. Proc. 1335, 728-735 (2011); doi: 10.1063/1.3591921

(C) 2011 American Institute of Physics 978-0-7354-0888-3/\$30.00 


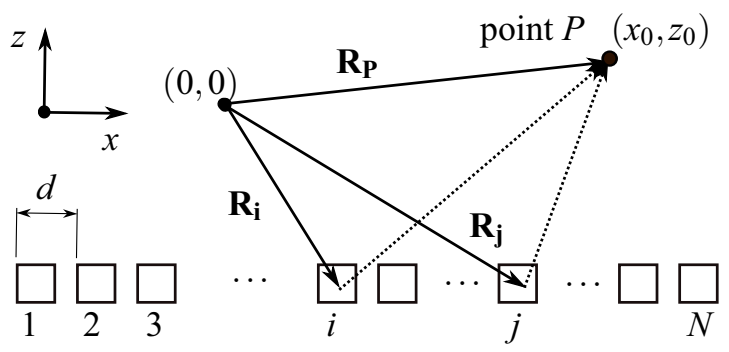

FIGURE 1. Linear array with $N$ square elements and pitch $d$, and its coordinate system.

the resulting image. One popular beamforming technique in SA is the dynamic focusing to compensate emission and reception delays simultaneously known as Total Focussing Method (TFM). Futhermore, SA post-processing allows to combine the TFM image with other information contained in the data to improve image quality[5].

In order to obtain different information levels from the same data set, several image compounding techniques have been proposed. Using broadband excitation and filtering, different images with frequency diversity, or from several frequency bands, can be obtained and combined [6]. Other approaches explore the effect of the apodization function. As it is wellknown, image sidelobe distribution is determined by the apodization function employed. If different apodization functions are applied to the same data set, it is possible to obtain slightly different images of the same scene that can be combined to remark coincidences. This strategy has been explored by several authors, for instance by selecting the minimum of two or more images from the same object [7]; with the use of constrained least squares techniques to minimize the energy of the point spread function (PSF) in certain boundaries and maintaining the peak at the focus, to improve contrast resolution [8]; or by means of a normalized cross-correlation function [9].

The signal phase is an additional information source to improve the image compounding technique. Based on the fact that the phase may carry more information in the location of events than the magnitude for certain classes of signals, some authors study the phase coherence between the array element signals in SA operation [10].

This work explores the diversity introduced in sidelobe distribution by different apodization functions, using the phase information as a discriminatory factor. The spatial diversity is increased by the use of two arrays positioned at different parts of the structure.

\section{SYNTHETIC APERTURE IMAGING}

Figure 1 shows a linear array with $N$ elements and pitch $d$ and its coordinate system. The beamforming technique employed in this work is the delay-and-sum (DAS), where all waveforms are focused at every point in the image:

$$
\operatorname{IM}\left(x_{0}, z_{0}\right)=\sum_{i=1}^{N} \sum_{j=1}^{N} v_{i j}\left(\tau_{i j}\left(x_{0}, z_{0}\right)\right),
$$

where

$$
\tau_{i j}\left(x_{0}, z_{0}\right)=\left(\left|\mathbf{R}_{\mathbf{i}}-\mathbf{R}_{\mathbf{P}}\right|+\left|\mathbf{R}_{\mathbf{P}}-\mathbf{R}_{\mathbf{j}}\right|\right) / c .
$$




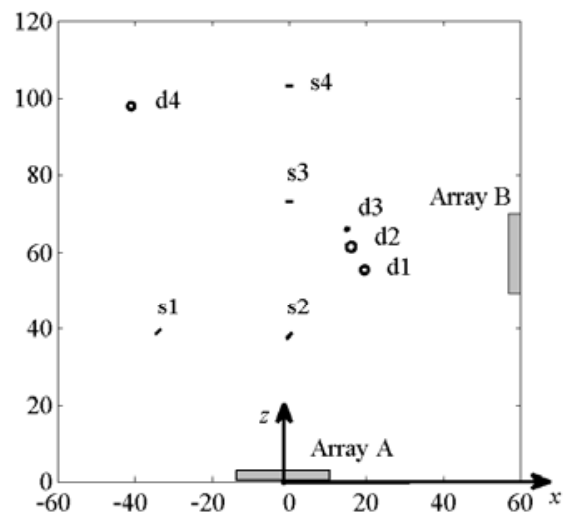

FIGURE 2. Artificial defects in an 1-mm-thick aluminum plate with dimensions $1200 \mathrm{~mm} \times 1200 \mathrm{~mm}$ : through-slits (s1 - s4) and bonded metal disks (d1 - d4). Dimensions in centimeters.

The image at every point $P\left(x_{0}, z_{0}\right)$ is obtained by the sum of all delayed combinations of transmitter-receiver pairs: $v_{i j}(t)$ is the signal transmitted by element $i$ and received by element $j ; \tau_{i j}\left(x_{0}, z_{0}\right)$ is the time delay from the transmitter at $\mathbf{R}_{\mathbf{i}}$ to the point $P$ and back to the receiver at $\mathbf{R}_{\mathbf{j}}$; and $c$ is the propagation velocity. Linear interpolation between samples is used in time domain to obtain the approximate signal amplitude values at the corresponding delays given by (2) when they are not an integer multiple of the sampling period.

\section{Experimental Setup}

An aluminum plate $(2024-\mathrm{T} 3), 1200 \mathrm{~mm} \times 1200 \mathrm{~mm}, 1-\mathrm{mm}$-thickness, was used as test piece. A linear array (called array A) was surface mounted at a border of the plate and another one (array B) was placed at an adjacent border of the plate, as seen in Fig. 2. Finally, several artificial defects were produced as through-slits (s1,s2,s3,s4) and bonded metal disks $(\mathrm{d} 1, \mathrm{~d} 2, \mathrm{~d} 3, \mathrm{~d} 4)$ at several locations and orientations.

A commercial 128-channel array system (SITAU-LF 32:128, DASEL S.L., Spain) excites the piezoelectric elements with programmable digital pulses with $-90 \mathrm{~V}$ amplitude, and digitizes the received waveforms (12 bits resolution, up to $40 \mathrm{MHz}$ sampling frequency), which are transferred to the computer for post-processing. Due to the nature of Lamb waves, the low dispersion $\mathrm{S} 0$ mode at $330 \mathrm{kHz}$ with propagation velocity of $5350 \mathrm{~m} / \mathrm{s}$ was selected for the experimentation.

The linear array has 30 elements and a pitch of $9 \mathrm{~mm}$. Each element of the array is a Pz-26, 0.5-mm-thickness $(7 \mathrm{~mm} \times 7 \mathrm{~mm})$ piezoelectric ceramic (Ferroperm, Denmark). For the SA experiments the array transducers were excited by a one-cycle square wave at the fundamental frequency of $330 \mathrm{kHz}$, and the RF data was acquired and stored for post-processing (bandpass filtered, interpolated and dynamically focused using (1) in a grid with spatial resolution of $0.5 \mathrm{~mm}$ ). The RF images are rectified and their envelope obtained after low-pass filtering with a two-dimensional spatial circular averaging filter that was designed in terms of spatial resolution and envelope extraction.

\section{Experimental Synthetic Aperture Images. Apodization}

Uniform and Blackman apodizations were chosen, because they produce images with 
(a)

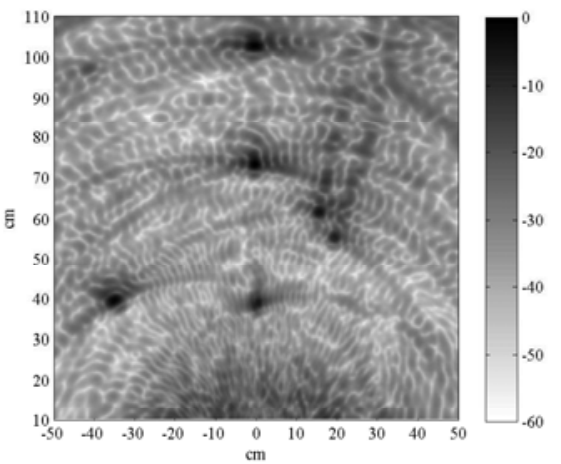

(c)

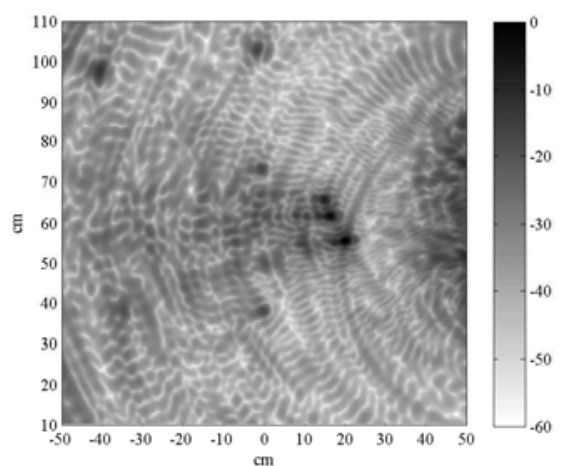

(b)

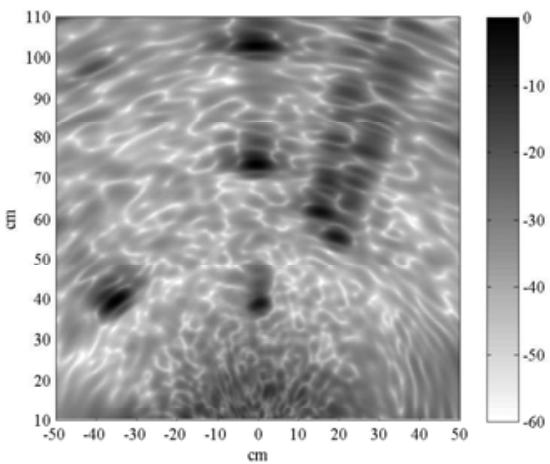

(d)

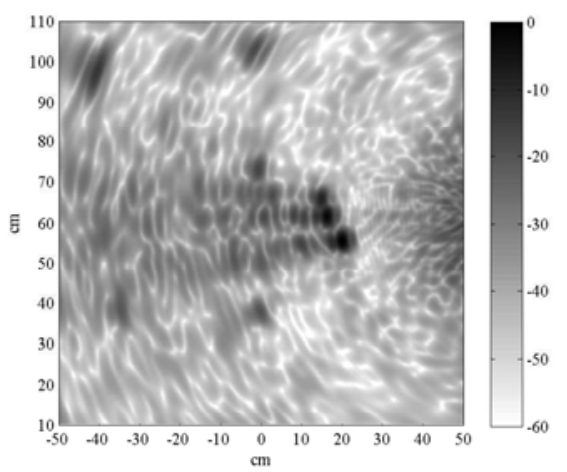

FIGURE 3. On top, conventional SA images for array A: (a) Uniform apodization, (b) Blackman apodization. On bottom, conventional SA images for array B: (c) Uniform apodization, (d) Blackman apodization.

considerable differences in the mainlobe width and the sidelobe levels. Figure 3 shows the resulting SA images obtained with array $\mathrm{A}$ and $\mathrm{B}$. The different apodizations result in different contrasts produced by the sidelobes and different resolutions produced by the finite mainlobe width. A deadzone close produced by the excitation pulse can be also observed close to the array, limiting the detection of defects in this region. Furthermore, there are reverberations behind the defects (mainly the surface defects, simulated by bonded disks) which indicate either false defects or shadows that could hide some weaker reflectors. For example, using the array $\mathrm{A}$, the disk $\mathrm{d} 3$ is not visualized because it is in the shadow of the larger disks. Likewise, the aluminum disk $\mathrm{d} 4$ has a lower level than the other brass disks in the image.

\section{Experimental Synthetic Aperture Images. Polarity Images}

The phase information of a B-mode image was presentad as the Phase or Signal Coherence Factor (SCF) in [10]. A polarity image can be formed at every point in the image from the sum of the polarity of the delayed RF waveforms. At a defect position all delayed signals should sum in phase, resulting in a high value (positive or negative) in the polarity image. When there is not a defect the phases would sum randomly and lower polarity signal levels are expected.

By considering $b_{i j}\left(x_{0}, z_{0}\right)$ the algebraic sign of the delayed RF data $v_{i j}$ (corresponding to a specific transmitter-receiver pair) at a particular position $\left(x_{0}, z_{0}\right)$ in the image, the polarity image at $\left(x_{0}, z_{0}\right)$ will be the normalized absolute value of the sum of $b_{i j}$ for all possible combinations of transmitter-receiver signals: 
(a)

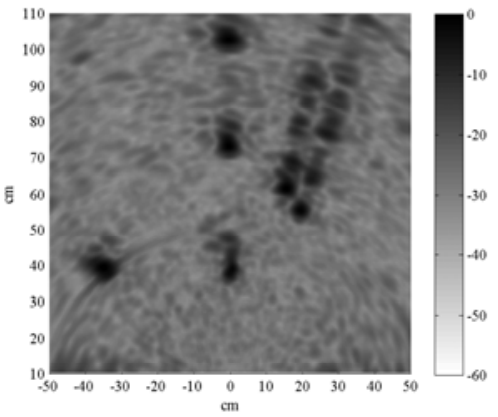

(b)

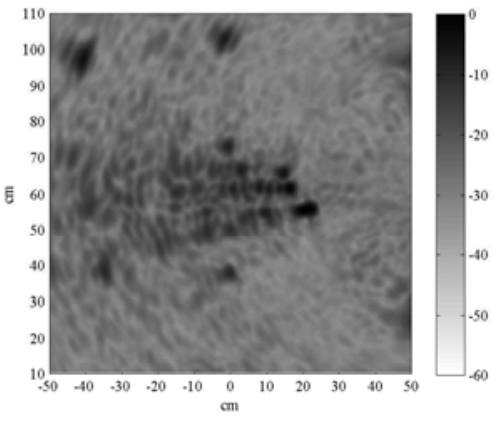

FIGURE 4. Envelope of the polarity image from (a) array A (b) array B.

$$
\operatorname{Pol}\left(x_{0}, z_{0}\right)=\left|\frac{1}{N^{2}} \sum_{i=1}^{N} \sum_{j=1}^{N} b_{i j}\left(x_{0}, z_{0}\right)\right|=\left|\frac{1}{N^{2}} \sum_{i=1}^{N} \sum_{j=1}^{N} \operatorname{sgn}\left\{v_{i j}\left(\tau_{i j}\left(x_{0}, z_{0}\right)\right)\right\}\right|,
$$

where $\operatorname{sgn}\{\cdot\}$ is the signum function:

$$
\operatorname{sgn}\{n\}=\left\{\begin{array}{ll}
+1, & n \geq 0 \\
-1, & n<0
\end{array} .\right.
$$

The envelope of the polarity image from array A and B is shown in Fig. 4. As we see, it does not depend on apodization. This polarity image still suffers the effects of the reverberations behind the defects, which sum in phase. However, the deadzone close to the array is practically eliminated, because there is not a coherent sum of the focused signals in this region. The polarity level indicates the presence of a defect, and this information, together with the different apodization images, is used in the algorithm proposed in the next section.

\section{MULTI-APODIZATION POLARITY TECHNIQUE}

The Multi-Apodization Polarity (MAP) technique is based on the combination of images obtained with different apodizations, which produce images with different defect resolution and contrast, using the phase or polarity of the delayed signals as a criterium for the image composition.

Figure 5 shows a block diagram of the proposed method where spatial diversity has been increased using both arrays. The input RF waveforms captured by the arrays are used to produce a set of images from the test piece: using different apodizations (Uniform and Blackman), and the polarity images. The RF polarity image is thresholded and then used as a decision variable to select, on a pixel-by-pixel analysis, a determined value among the SA images. The thresholding operation is based on the observation that, at a defect, the polarity image will present higher absolute values due to the coherent sum of the delayed RF signals.

Figure 6 is used to explain the MAP technique, showing the expected effect when it is applied to a line of the image. The curves in Fig. 6(a) and 6(c) correspond to the absolute values of the pixels in a horizontal line in the image over a through-slit defect (s3), which is seen by array A and obtained with Uniform and Blackman apodizations, respectively. The normalized polarity function is presented in Fig. 6(b), and a threshold level of $70 \%$ is assumed (horizontal dotted line). When the polarity signal is above the threshold, the maximum of the absolute value from both apodization images is selected, in this case the Uniform one. This 


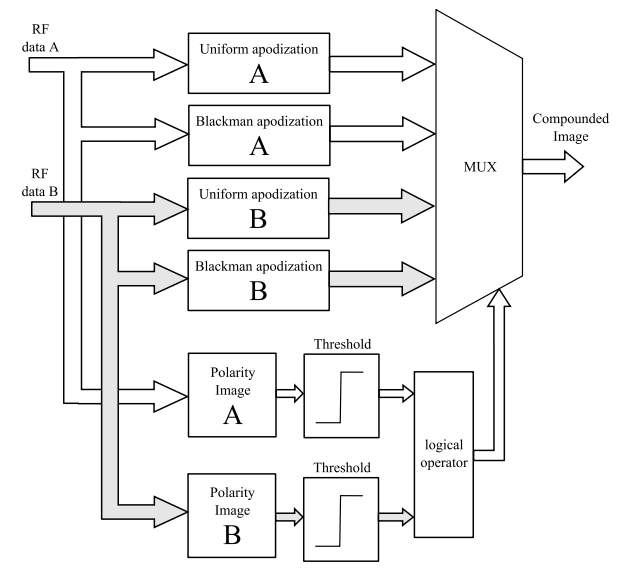

FIGURE 5. Block diagram of the Multi-Apodization Polarity technique with spatial devirsity.

(a)

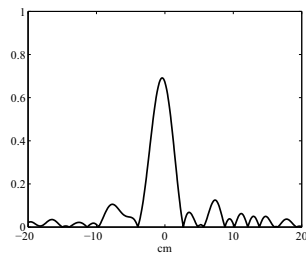

(c)

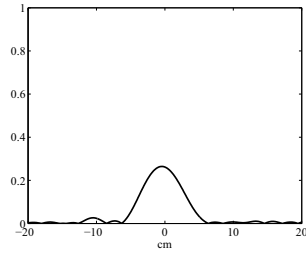

(b)

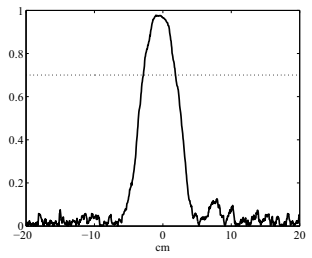

(d)

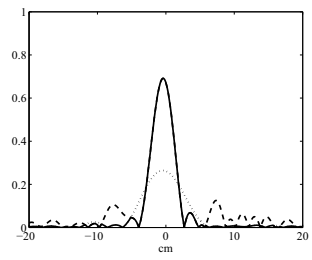

FIGURE 6. Example of the MAP technique for a line (absolute values) in the image containing a through-slit defect. (a) From Uniform apodization; (b) Polarity and threshold level of 70\% (dotted line); (c) From Blackman apodization; (d) Compounded result (solid line) and the two apodization images for comparison.

will preserve the narrow mainlobe resulting from the Uniform apodization. Below threshold, the minimum value is selected, which depends on the different apodizations and reflectors characteristics. The compounded result is represented by the solid line curve in Fig. 6(d), and the two apodization image lines are also shown for comparison. The narrower mainlobe from the Uniform apodization is preserved, whereas the sidelobes levels are reduced, as expected. The compounded image is then smoothed by an averaging filter to obtain the envelope images.

A logical operator OR has been chosen to combine both images. Thus, there is a high probability of detection of a defect, although it would be detected by only one of the arrays. Under this condition, the maximum of the absolute values of the four SA images is selected. Otherwise the minimum is selected.

The threshold level is the parameter that controls the sensitivity of the method. Figure 7 shows images obtained with the MAP technique (left), and the corresponding binary thresholded-ORed polarity images (right) for different threshold levels of $60 \%$ (top), $70 \%$ (middle) and 90\% (bottom). The dark regions in Fig. 7(b), 7(d) and 7(e) indicate the polarity image points from array A or array B that are above threshold. By using lower threshold values the resolution is a bit lower and the upper-left defect (disk d4) is better visualized, but false defects due to the reverberations close to defects $\mathrm{d} 1 \mathrm{-d} 3$ start to appear. Higher threshold levels reduce detectability of smaller or weaker reflectors. However, even when the polar- 
(a)

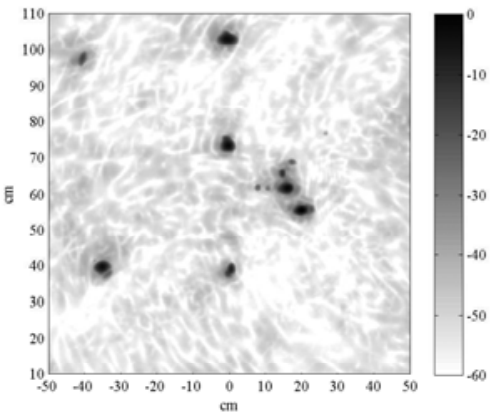

(c)

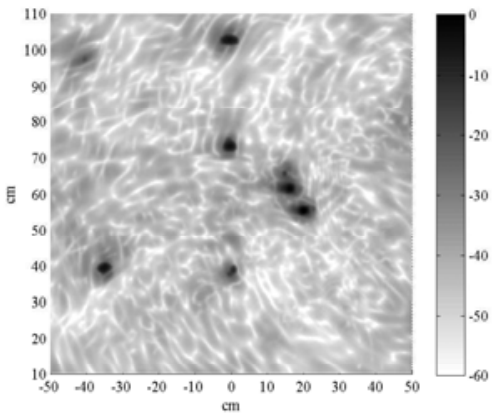

(e)

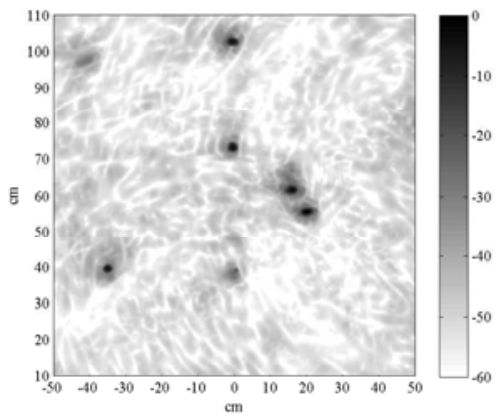

(b)

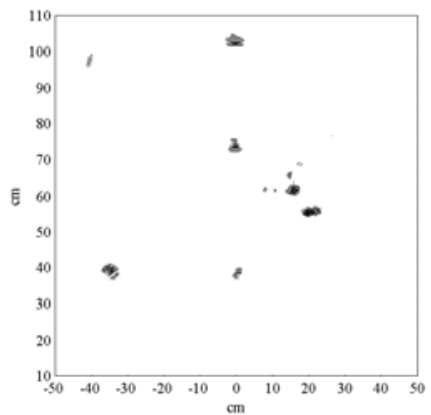

(d)
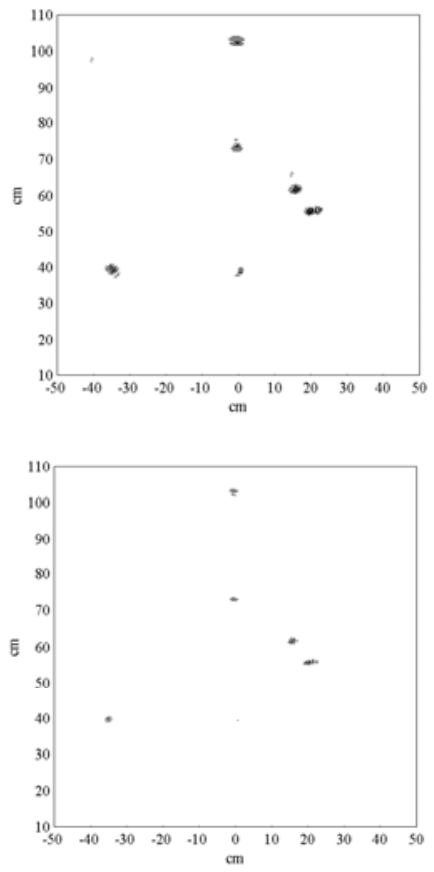

FIGURE 7. MAP images (left) and thresholded-ORed polarity images (right) for different threshold levels. (a) and (b) $60 \%$; (c) and (d) 70\%; (e) and (f) $90 \%$.

ity images are below the threshold in the presence of a defect, or give a weak evidence of a defect, the proposed image compounding technique can still result in its detection. This effect can be seen, for example, when the threshold level is $90 \%$, where there are not indications of defect $\mathrm{s} 2$ in the thresholded-ORed polarity image in Fig. 7(f), but it appears in the compounded amplitude image in Fig. 7(e).

\section{CONCLUSIONS}

The MAP technique, involving image compounding of two or more images from the same object obtained from different apodizations and arrays, and with the selection rule from the signal phases or polarities, results in high resolution defect sizing and location, as well as improved contrast. The polarity image has been presented as a valuable tool to indicate the presence of defects. The positioning of the arrays relative to the defects is very important and introduces spatial diversity, because a defect that is hidden for one of the arrays could be visible by other. The technique has been succesfully tested in isotropic plates and using non-dispersive single mode Lamb waves. 


\section{ACKNOWLEDGMENTS}

This work was supported in part by the Spanish Ministry of Science and Innovation under the projects DPI2007- 65408-C02-01, DPI2007-65408-C02-02, and PSE-020000-200910 (PSS-020000-2009-25 subproject) co-supported by ERDF (European Regional Development Fund). R. T. Higuti thanks the Ministry of Education of Brazil (Capes) for a postdoctoral scholarship (3711-08-0), which was developed at CSIC in 2009.

\section{REFERENCES}

1. D. E. Chimenti, Applied Mechanics Reviews 50, 247 (1997).

2. P. Wilcox, M. Lowe and P. Cawley, IEEE Transactions on Ultrasonics, Ferroelectrics and Frequency Control 52, 653 (2005).

3. K. Diamanti, C. Soutis and J. M. Hodgkinson, Composites Part A 38, 1121 (2007).

4. J. Vishnuvardhan, A. Muralidharan, C. V. Krishnamurthy and K. Balasubramaniam, NDT\&E International 42, 117 (2009).

5. P. D. Wilcox, C. Holmes and B. W. Drinkwater, IEEE Transactions on Ultrasonics, Ferroelectrics and Frequency Control 54, 1541 (2007).

6. Jennifer E. Michaels and Thomas E. Michaels, Wave Motion 6, 482(2007).

7. H. C. Stankwitz, R. J. Dallaire and J. R. Fienup, IEEE Transactions on Aerospace and Electronic Systems 31, 267(1995).

8. D. A. Guenther and W. F. Walker, IEEE Transactions on Ultrasonics, Ferroelectrics and Frequency Control 54, 332(2007).

9. C. H. Seo and J. T. Chen, IEEE Transactions on Ultrasonics, Ferroelectrics and Frequency Control 55, 2198 (2008).

10. J. Camacho, M. Parrilla and C. Fritsch, IEEE Transactions on Ultrasonics, Ferroelectrics and Frequency Control 56, 958 (2009). 
Copyright of AIP Conference Proceedings is the property of American Institute of Physics and its content may not be copied or emailed to multiple sites or posted to a listserv without the copyright holder's express written permission. However, users may print, download, or email articles for individual use. 